\title{
Normalisierung der geschlechtlichen Entwicklung kopfloser Fragmente junger 우오 von Platynereis dumerilii (Polychaeta) durch Behandlung mit konservierten Prostomien juveniler Individuen
}

\author{
C. Hauenschild \\ Zoologisches Institut der Technischen Universität Braunscbweig; \\ Braunschweig, Bundesrepublik Dewtschland
}

\begin{abstract}
Normalization of sexual development in headless fragments of young female Platynereis dumerilii (Polychaeta) due to treatment with preserved prostomia of juvenile specimens. Fragments taken from female Platynereis dumerilii are, at an early stage, characterized by small oocytes, unable to develop normal eggs and to complete the epitokous state if deprived of hormone supply from the prostomium. The question remained whether this inability to achieve normal sexual maturity is due to complete lack of common "juvenile brain hormone" or to absence of a special phase-specific "maturation hormone" whose existence has not yet been proved, but suggested on the basis of autoradiographic investigations of neurosecretory brain activity (MUjLLER 1973). New experiments on such fragments demonstrate that fairly normal oogenesis can be induced and a more advanced epitoke formation attained by daily external treatment with dried isolated prostomia taken from juvenile specimens. Thus, there is no need to assume interference of a special "maturation hormone" in sexual development. The treated fragments show normal posterior regeneration followed by fairly normal sexual maturation, even if the supply of "juvenile brain hormone" is not changed throughout the experiment; hence it may be postulated that the fragment itself gradually alters its mode of response to the hormone.
\end{abstract}

\section{EINLEITUNG UND FRAGESTELLUNG}

Im Anschluß an Befunde, die Durchon $(1948,1952)$ an natürlich aufgewachsenen ¿ $\delta$ mehrjähriger Nereis-Arten erhoben hatte, stellte ich bei den im Labor gezüchteten 9 우 von Platynereis dumerilii ebenfalls eine Beeinflussung der geschlechtlichen Entwicklung durch ein im Prostomium erzeugtes Hormon ("Gehirnhormon ") fest (Hauenschild 1956, 1966). P. dumerilii ist eine getrenntgeschlechtliche, mit 25-40 mm ausgewachsene Art, die durchschnittlich in einem Alter von $1 / 2 \mathrm{bis} 3 / 4 \mathrm{Jahr}$, frühestens mit 3 Monaten, geschlechtsreif wird (bei $20^{\circ} \mathrm{C}$ ) und sich nach einer epitoken Metamorphose von ca. 1 Woche Dauer als freischwimmende "Heteronereis" fortpflanzt (Abb. 1). Während Durchon nach experimenteller Entfernung der Hormonquelle bei den unreifen $\delta \delta$ von Perinereis cultrifera einen vorzeitigen Eintritt aber dennoch qualitativ 
normalen Verlauf der Spermatogenese und der epitoken Metamorphose beobachten konnte und daraus auf eine die geschlechtliche Entwicklung lediglich hemmende Funktion des Gehirnhormons schloß, ergaben die Versuche mit den 오우 von $P$. dumerilii differenziertere Ergebnisse (vergl. auch Hauenschind 1974):

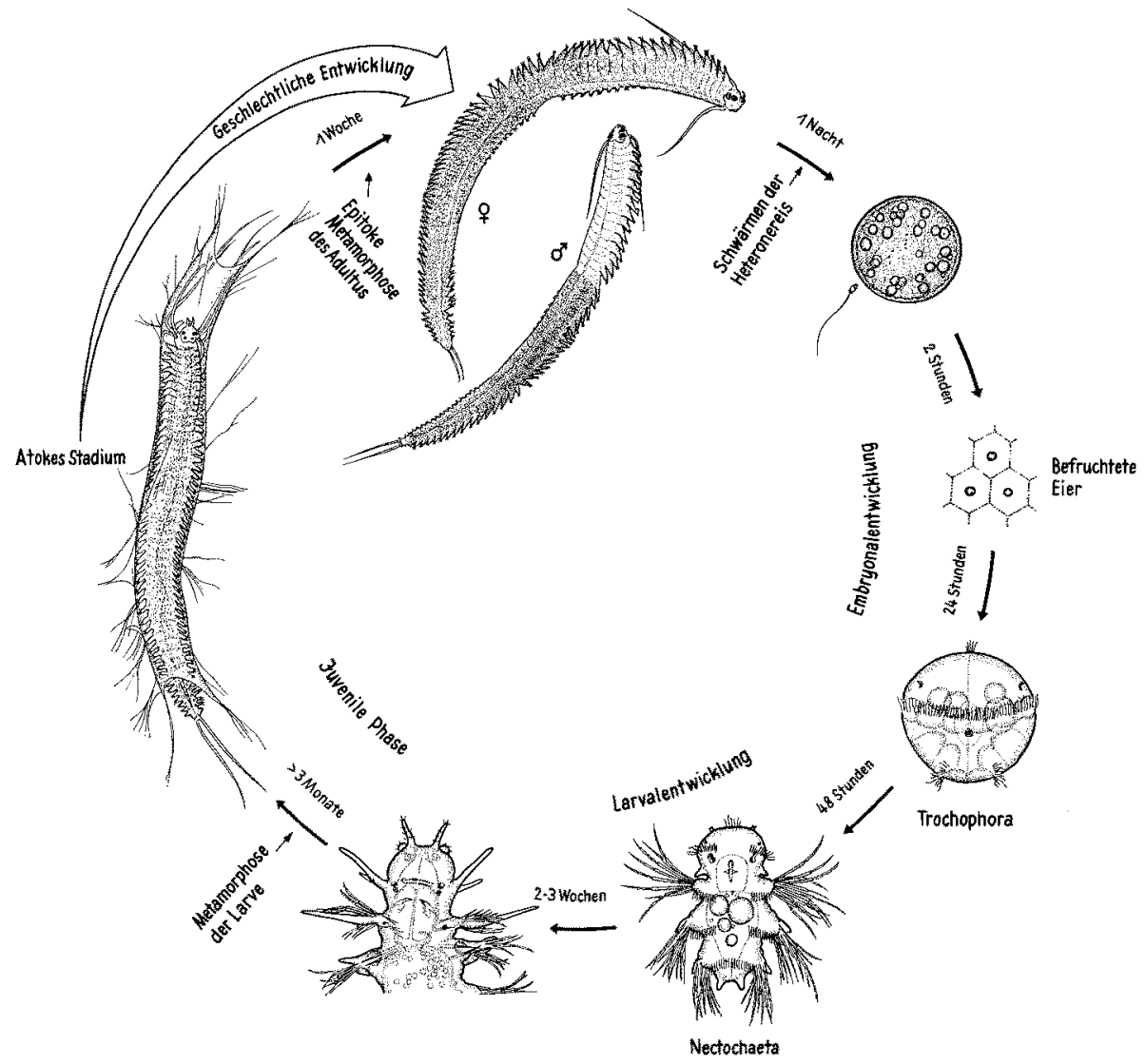

Entwicklungszyklus von Platynereis dumerilii

Abb. 1: Die wichtigsten von einer Platynereis dumerilii während ihres Lebens durchlaufenen Stadien in ihrer auf $20^{\circ} \mathrm{C}$ bezogenen zeitlichen Abfolge. (Einzelfiguren z. T. nach HAUENSCHILD \& FisChER 1969)

Nur $q q$ oberhalb eines "kritischen Stadiums", deren Oocyten bereits einen Durchmesser von mehr als $100 \mu$ m erreicht haben (Abb. $7 \mathrm{~b}$ ), reagieren auf eine Abtrennung des Prostomiums mit einer beschleunigt, aber qualitativ \pm normal vollzogenen sexuellen Entwicklung, in deren Verlauf die Oocyten zu funktionsfähigen Eiern mit einem Durchmesser von 160-180 $\mu \mathrm{m}$ heranwachsen (Abb. 11b) und die Parapodien (Pp) alle epitoken Merkmale vollständig ausbilden (Abb. 9); dagegen bewirkt die Entfernung der Hormonquelle bei jüngeren $q Q$ (z. B. mit Oocyten zwischen 30 und $60 \mu \mathrm{m}$ ) nur eine von gestörter Vitellogenese begleitete rasche Größenzunahme der Oocyten, aus der 
jedoch lediglich funktionsunfähige Eizellen ohne die für reife Eier typische subkortikale Mucopolysaccharid-Schicht resultieren ( $\mathrm{Abb} .7 \mathrm{a}$ ) sowie eine nur angedeutete epitoke Entwicklung, die in ein abnormes, etwa 2 Wochen nach Enthirnung absterbendes Endstadium mündet ( $\mathrm{Abb} .5$ und 6 ). Solche 99 , die das kritische Stadium bei Wegnahme ihres Prostomiums noch nicht erreicht haben, können aber - auch als kurze, nur aus wenigen Segmenten bestehende Fragmente - normal überleben und zu einem späteren Zeitpunkt durchaus vollwertig geschlechtsreif und epitok werden, wenn ihnen innerhalb weniger Tage nach Trennung von ihrem eigenen Prostomium dieses oder das abgeschnittene Prostomium eines anderen Individuums ins Coelom implantiert wird. Diese Befunde ließen zunächst die Möglichkeit offen, daß das Prostomium nacheinander zwei verschiedene Hormone produziert, nämlich zuerst nur ein die geschledtliche Entwicklung hemmendes "juveniles Hormon“, später mit Annäherung an das kritische Stadium aber zunehmend ein entgegengesetzt wirkendes "Reifungshormon", ohne dessen Mitwirkung eine normale Eireifung und Epitokie nicht möglich ist. Die Ergebnisse weiterer Experimente sprachen jedoch gegen die Annahme eines besonderen Reifungshormons, ohne freilich die Existenz eines solchen gewissermaßen gonadotropen Prinzips mit letzter Sicherheit ausschließen zu können; es handelt sich dabei vor allem um Versuche, in denen eine vorzeitige aber qualitativ normale geschlechtliche Reifung von "vorkritischen" 우 (Oocyten 30-50 $\mu \mathrm{m}$ ) entweder durch eine genau dosierte UVBestrahlung des Gehirns oder mittels Ersatz des eigenen Prostomiums durch den aufgepfroptten Kopf eines 10-20 Tage alten, noch larvalen Spenders ausgelöst werden konnte (Haunnschild 1966). Diese und andere Befunde führten zu der Hypothese, daß die im Verlauf der Entwicklung abnehmende Konzentration eines einzigen, qualitativ unverändert bleibenden Gehirnhormons (d. h. des juvenilen Hormons) die zeitliche Abfolge der geschlechtlichen Reifungsvorgänge steuert, indem jeder dieser Prozesse nur bei einem ganz bestimmten Hormonspiegel in Gang gesetzt wird (Hauenschim 1965); die Annahme eines solchen Mechanismus vermag formal zu erklären, warum im vorkritischen Stadium eine normale geschlechtliche Entwicklung nur möglich ist, wenn die Hormonausschüttung allmählich gedrosselt wird und der Hormontiter auf jedem Niveau genügend lange verharrt, um die daran geknüptten Entwicklungsvorgänge auslösen zu können, nicht aber, wenn die Hormonquelle abrupt entfernt und der Hormonspiegel dadurch sehr rasch bis auf Null abgesenkt wird. Durchon \& PORCHET (1971) haben durch Bestimmung der im Gehirn von Perinereis cultrifera und anderen Nereiden in verschiedenen Entwicklungsstadien vorhandenen Hormonmenge mit Hilfe eines biologischen Tests die genannte Hypothese erhärtet; sie fanden, daß der Gehirnhormon-Spiegel im Verlauf der geschlechtlichen Reifung tatsächlich stufenweise und zum Schluß bis auf Null absinkt und daß dabei jedem Hormon-Konzentrationsbereich ein cytologisch oder histochemisch definiertes Stadium der Oo- bzw. Spermatogenese zugeordnet werden kann. Schon früher hatten dieselben Autoren festgestellt, daß die heranwachsenden Eier auf die inkretorische Aktivität des Gehirns im Sinne einer Mitkoppelung hemmend zurückwirken (Durchon 1952, Porchet 1967); da 9 \% von $P$. dumerilii, die infolge Hungers überhaupt keine Eier im Coelom haben, eine durchaus normale epitoke Metamorphose durchmachen können, scheint aber eine solche Beeinflussung des Gehirns durch die Geschlechtsprodukte für die sexuelle Reifung keineswegs obligatorisch zu sein. 
Außer für die normale geschlechtliche Entwicklung ist das Inkret des Prostomiums auch für die teloblastische Neubildung von Segmenten unbedingt erforderlich; kopflose Fragmente bilden nur dann ein caudales Regenerat von normalem Umfang und Differenzierungsgrad, wenn ihnen Gehirnhormon, z. B. durch Implantation eines lebenden Prostomiums ins Coelom, in ausreichender Menge zugeführt wird (Hauenschmo 1960). Da sich nach den bisherigen Ergebnissen die Neubildung von Segmenten auf der einen und eine rasche geschlechtliche Entwicklung auf der anderen Seite wechselseitig strikt ausschließen, liegt eine Abhängigkeit beider Vorgänge von ein und demselben Hormon nahe; dabei wurde bisher meist angenommen, daß viel Hormon die Regeneration, wenig Hormon dagegen die geschlechtliche Reifung fördert.

Die Bildung des vom Nereiden-Prostomium abgesonderten Hormons wird seit langem neurosekretorischen Zellen (NSZ) im Gehirn zugeschrieben, ohne daß bisher allerdings eine differenzierte Zuordnung experimenteller und cytologischer Befunde möglich war. Neuerdings hat MüLler (1973) den Verlauf der Neurosekretion im Gehirn von $P P$. dumerilii in verschiedenen Stadien der geschlechtlichen Entwicklung autoradiographisch untersucht und entgegen den Erwartungen im Verlauf der geschlechtlichen Reifung keine Abnahme, sondern in zwei Bereichen des Gehirns eine drastische Zunahme des Bestandes an großen, synthetisch aktiven NSZ gefunden. Da diese Zellen, wie Kontrollversuche gezeigt haben, ihr Neurosekret nicht speichern, sondern laufend abgeben, kann ihr Produkt nicht juveniles Hormon sein; denn dieses wird, wie experimentell nachgewiesen ist, im Verlauf der geschlechtlichen Reifung immer weniger und schließlich gar nicht mehr ausgeschüttet, d. h. seine Produktion verhält sich genau reziprok zu der autoradiographisch faßbaren Zunahme der neurosekretorischen Aktivität. Unter dem Eindruck der auffälligen zeitlichen Koinzidenz des Erscheinens zusätzlicher NSZ mit dem Fortgang der geschlechtlichen Reifung drängte sich daher der Verdacht auf, daß die betreffenden, erst während der Reifung in zunehmender Anzahl in Erscheinung tretenden Zellen vielleicht doch ein besonderes Reifungshormon erzeugen, obwohl ein solches experimentell bisher nicht nachgewiesen werden konnte. Erhärtet wurde der Verdacht durch den Befund MüLLERs, daß die zusätzlichen NSZ im Gehirn reifender Tiere wieder verschwinden, wenn man durch Amputation eines umfangreichen hinteren Rumpfabschnitts eine Regeneration erzwingt, wobei automatisch der Fortgang der sexuellen Entwicklung für die Dauer des Regenerationsprozesses blockiert wird. Diese Feststellung macht gleichzeitig deutlich, daß sich mit der bisher angewendeten Methode der Transplantation lebender Prostomien eine eventuell von diesen zusätzlichen NSZ ausgehende besondere Hormonwirkung grundsätzlich nicht nachweisen läßt, weil diese Prostomien auf eine ihrer Transplantation notwendigerweise vorausgehende Abtrenunng vom reifenden Spender wie im Fall der geschilderten Rumpfamputation mit Rückbildung eben dieser NSZ reagieren müssen.

Damit war die Frage wieder aktuell: Produziert das Nereiden-Gehirn außer dem experimentell nachgewiesenen juvenilen Hormon, welches die geschlechtliche Entwicklung hemmt bzw. verzögert, in der Endphase der Ontogenie zusätzlich oder stattdessen noch ein anderes, entgegengesetzt wirkendes Reifungshormon, welches eine normale Oogenese und Epitokie überhaupt erst ermöglicht? Eine Methode zur endgültigen Klärung des Sachverhalts schien mir darin zu bestehen, daß Fragmenten von $q$ 우 im vorkritischen Stadium, die ja ohne Hormonzufuhr keine normale geschlechtliche Entwick- 
lung durchlaufen können, täglich Hormon aus abgetöteten Prostomien appliziert wird, welche entweder von entsprechend jungen Individuen oder von in ihrer Reifung \pm weit fortgeschrittenen $q O$ stammen; im Gegensatz zur einmaligen Implantation eines lebenden Prostomiums, das seine im Coelom des Wirtsfragments andauernde Hormonproduktion im Lauf des Versuchs nicht nur quantitativ, sondern auch qualitativ ändern könnte, wäre durch ein solches Verfahren sichergestellt, daß das Testfragment während der gesamten Versuchsdauer stets das gleiche Hormon in einer definierten Menge zugeführt bekommt. Sollte sich dann z. B. herausstellen, daß ein Q-Fragment mit Oocyten von $40 \mu \mathrm{m}$ bei ausschließlicher Zufuhr von juvenilem Hormon aus geschlechtlich noch undifferenzierten Spendern entwicklungsfähige Eier und normale epitoke Merkmale hervorzubringen vermag, wäre damit die Notwendigkeit eines besonderen, nur im fortgeschrittenen Stadium gebildeten Reifungshormons für die geschlechtliche Entwicklung auszuschließen. Ob dies zutriff, werden die im folgenden beschriebenen Versuche zeigen.

\section{MATERIAL UND METHODE}

Alle in den Versuchen und als Kontrollen verwendeten Testfragmente stammten, ebenso wie auch die konservierten Prostomien, ausnahmslos von Tieren, die in meinen seit mehr als 20 Jahren in kontinuierlicher Generationenfolge gehaltenen Laborzuchten von Platynereis dumerilii vom $\mathrm{Ei}$ an aufgezogen worden waren und deren Vorgeschichte deshalb gut bekannt ist. Abweichend von der bei HAuENSCHILd \& Fischer (1969) beschriebenen Kulturmethode werden die heranwachsenden Würmer, sobald sie 6 Wochen alt sind, seit einigen Jahren mit einer Mischung aus tiefgekühltem Spinat und Zierfischfutter ernährt. Als Spender für die Testfragmente dienten 8-12 Monate alte 우, die bei einer Länge von $24-31 \mathrm{~mm}$ aus $65-71$ mit $\mathrm{Pp}$ bestückten Segmenten (PpS) bestanden und deren Oocyten im Durchmesser maximal 35-40 $\mu \mathrm{m}$ maßen; für die Längenmessung, für die Bestimmung der Oocytengröße an Hand einer aus dem Coelom entnommenen Probe und für das Herausschneiden der Fragmente bzw. Prostomien wurden die Tiere in einer Mischung aus 1 Teil Seewasser und 2 Teilen isotonischer $\mathrm{MgCl}_{2}$-Lösung narkotisiert. Die Fragmente bestanden meist aus $5 \mathrm{PpS}$ und entstammten ausschließlich dem Bereich zwischen 22. und 36. PpS, d. h. der vorderen praesumptiv epitoken Region; am ersten Tag nach ihrer Isolierung wurden sie zwecks Erleichterung des Wundverschlusses in antibiotischem Seewasser (AS) gehalten (synthetisches Seewasser mit $150 \mathrm{mg}$ Streptomycin und $75 \mathrm{mg}$ Penicillin pro Liter).

Das zum Versuch bestimmte Fragment wurde vom 2. oder 3. Tag nach seiner Isolierung an täglich aufs neue in einem kleinen, in einer feuchten Kammer hängenden Tropfen aus AS mit 3 abgetöteten juvenilen Prostomien zusammengebracht (Abb. 2). Die besten Resultate ergab eine intermittierende Behandlung, bei der das Fragment jeden Tag nur 8 Stunden lang in dem mit neuen Prostomien versehenen AS-Tropfen (ca. 5-10 $\mu$ l) untergebracht und dabei dem aus den Prostomien herausgelösten Gehirnhormon ausgesetzt war, während es sich in den übrigen 16 Stunden in einer Boverischale mit $10 \mathrm{ml}$ normalem Seewasser, welches keine Prostomien enthielt, von dieser Behandlung erholen konnte; meist befand sich das Versuchsfragment von 9-17 Uhr im 
hängenden Tropfen mit den 3 Prostomien und von 17-9 Uhr in der Boverischale. Die als juvenil bezeichneten Prostomien wurden von jungen, aus weniger als 50 PpS bestehenden Tieren gewonnen; in diesem Stadium sind noch keine frei im Coelom flottierenden Geschlechtsprodukte nachweisbar, weshalb das Geschlecht der Prostomien-Spender unbekannt blieb. Die in Narkose entlang dem Hinterrand des Prostomiums abgetrennten Köpfe wurden, nachdem ihnen die Palpen und die peristomialen Anteile einschließlich der Fühlerzirren entfernt worden waren, kurz in Aqua dest. abgespült, auf einem Streifen Filtrierpapier im Exsiccator getrocknet und anschließend bis zum Ge-

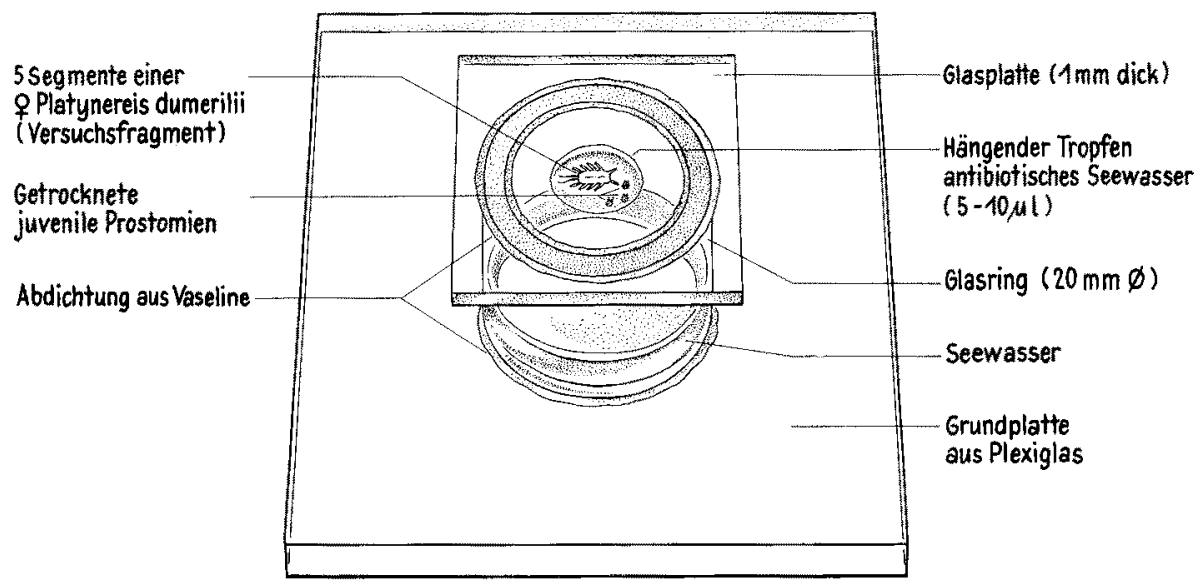

Abb. 2: Versuchsanordnung zur Behandlung eines Q-Fragments mit dem in 3 getrockneten Prostomien geschlechtlich noch undifferenzierter Spender enthaltenen Gehirnhormon

brauch (höchstens 6 Wochen lang) bei $-20^{\circ} \mathrm{C}$ aufbewahrt. Jeden Tag um 9 Uhr wurden 3 von diesen tiefgekühlten Prostomien auf die Versuchstemperatur von $+20^{\circ} \mathrm{C}$ $\left( \pm 0,5^{\circ} \mathrm{C}\right.$ ) erwärmt und zusammen mit dem Versuchsfragment in einen kleinen Tropfen AS auf ein Glasplättchen gebracht, welches umgedreht und mit nach unten hängendem Tropfen auf den mit Vaseline bestrichenen Rand einer feuchten Kammer gelegt wurde (Abb. 2). Wenn die Kammer 8 Stunden später geöffnet und das Fragment nach gründlichem Abspülen in eine Boverischale mit frischem Seewasser übertragen wurde, waren die Prostomien, die natürlich kein zweites Mal verwendet werden konnten, bereits stark in Auflösung begriffen; ausgeprägte Fäulnisprozesse wurden aber bis zu diesem Zeitpunkt durch die zugesetzten Antibiotika ausreichend unterdrückt. Durch seine Bewegungen sorgte das Versuchsfragment für eine gleichmäßige Verteilung der aus den Prostomien in den Tropfen übertretenden Substanzen.

Als Kontrolle, die nicht mit Prostomien versehen, aber ansonsten genauso behandelt wurde, diente entweder ein dem Versuchsfragment benachbartes, 5 PpS umfassendes Stück des gleichen Spenders oder das topographisch genau entsprechende Fragment eines anderen, hinsichtlich Größe und Entwicklungsstadium vergleichbaren $q$; in einigen Versuchen gab es Kontrollen sowohl von der einen als auch von der anderen Sorte. 


\section{ERGEBNISSE}

Es liegen 11 Versuche an $q 9$, in denen jeweils ein Fragment mit dem Gehirnhormon aus juvenilen Prostomien äußerlich behandelt wurde, und die dazugehörigen unbehandelten Kontrollen vor. Von einigen technisch bedingten Ausfällen bzw. partiellen Mißerfolgen abgesehen, erbrachten die Experimente ein prinzipiell übereinstimmendes Ergebnis; es soll im folgenden an dem konkreten Fall des Versuchs vom 29. 10. 1973, dessen Verlauf als repräsentativ gelten kann, im einzelnen dargestellt werden.

Das die PpS 22-26 umfassende Versuchsfragment (VF) und das aus dem 27.-31. PpS bestehende Kontrollfragment (KF) entstammte ein und demselben $O$; dieses war zum Zeitpunkt seiner Zerstïckelung, der im folgenden als 1. Versuchstag (= Tag) gezählt werden soll, $8^{1 / 2}$ Mcnate alt und hatte eine Länge von $31 \mathrm{~mm}$, eine Segmentzahl von $71 \mathrm{PpS}$ sowie Oocyten, die maximal $40 \mu \mathrm{m}$ groß und noch aggregiert waren. Am 3. Tag kamen die Fragmente zum erstenmal für 8 Stunden in den kleinen, in der feuchten Kammer hängenden Tropfen, wobei dem VF 3 getrocknete juvenile Prostomien zugesetzt wurden. Das VF erhielt dann fortlaufend bis zum 19. Tag, also 17 Tage lang, täglich für die Dauer seines 8stündigen Aufenthalts im Tropfen je 3 neue juvenile Prostomien, wohingegen das KF während seiner ganzen Oberlebenszeit von jeglicher Gehirnhormon-Zufuhr abgeschnitten war. Am 3. Tag sahen VF und KF noch genau gleich aus und zwar, abgesehen von dem inzwischen erfolgten Wundverschluß, ebenso wie zu Versuchsbeginn (vgl. Abb. 3); ihre Pp waren völlig atok (vgl. Abb. 4) und beide Sorten von tiefliegenden Chromatophoren noch vollzählig erhalten. Bereits am 4. Tag waren am Hinterende von VF und KF knopfförmige Uritenanlagen zu erkennen; am 5. Tag hatte sich bei beiden die Anlage eines Regenerationskegels gebildet und beim VF, nicht jedoch beim KF, waren kurze Uriten ausgewachsen. Am 6. Tag begannen beim KF, wie in Abwesenheit des Gehirnhormons üblich, die ersten Chromatophoren zu degenerieren, wohingegen sie beim VF unverändert komplett erhalten waren. Von nun an unterschied sich der Habitus von VF und KF von Tag zu Tag deutlicher, und es war bereits erkennbar, daß von den konservierten Prostomien wirksames Gehirnhormon in den Tropfen abgegeben und vom VF aus ihm, vermutlich durch das Integument, aufgenommen wurde.

Beim Versuchsfragment waren am 7. Tag die Uriten vollständig ausgewachsen, der Regenerationskegel hatte sich gestreckt und ein erstes Paar kleiner regenerierter $\mathrm{Pp}$ wurde an ihm sichtbar; diese Neubildung hinterer Segmente setzte sich, und zwar in qualitativ völlig normaler Weise, bis zum 10. Tag fort, an dem das VF schließlich ein aus 3 voll differenzierten Pp-Paaren und 1 Paar Pp-Anlagen bestehendes Regenerat aufzuweisen hatte (Abb. 10). Eine solche Regenerationsleistung läßt, $\mathrm{da}$ sie ohne Prostomium niemals erbracht wird, mit Sicherheit auf die Mitwirkung eines gewissen Quantums Gehirnhormon schließen, welches im vorliegenden Fall nur aus den zugesetzten Prostomien in den Tropfen übergetreten und von außen in das VF eingedrungen sein konnte. In quantitativer Hinsicht blieb die Regenerationsleistung allerdings hinter derjenigen eines entsprechenden, aber mit einem lebenden Prostomium im Coelom ausgestatteten Fragments, das mindestens 5-6 PpS regeneriert hätte, deutlich zurück; angesichts der äußerlichen und auf täglich 8 Stunden beschränkten Applikation des Hormons und seiner nicht unerheblichen Verdünnung durch die 5-10, 1 Außen- 
medium erscheint dies jedoch kaum verwunderlich. Am 10. Tag, als seine caudale Regeneration zum Stillstand kam, war das VF, abgesehen von dem nunmehr allmählich einsetzenden Verlust seiner tiefliegenden Chromatophoren, noch vollständig atok

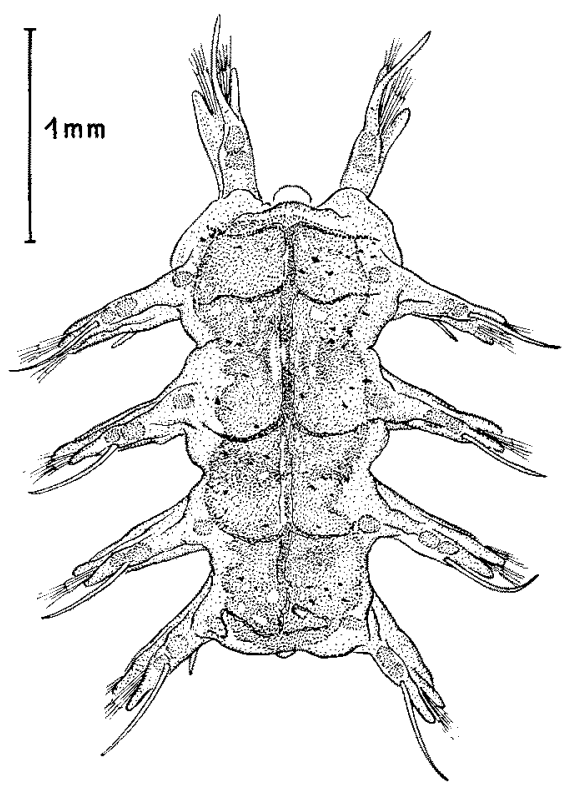

Abb. 3: Aus 5 Segmenten bestehendes Fragment (32.-36. PpS) eines $q$ mit $40 \mu \mathrm{m}$ großen Oocyten am 2. Versuchstag. Abgesehen vom Verschluß der Wundflächen, der seinerseits die unnatürliche Vorwärtsdrehung des vordersten Parapodienpaares bewirkt, sehen die Segmente noch genauso aus wie unmittelbar vor oder nach der 24 Stunden zurückliegenden Abtrennung der übrigen Teile des Spendertieres

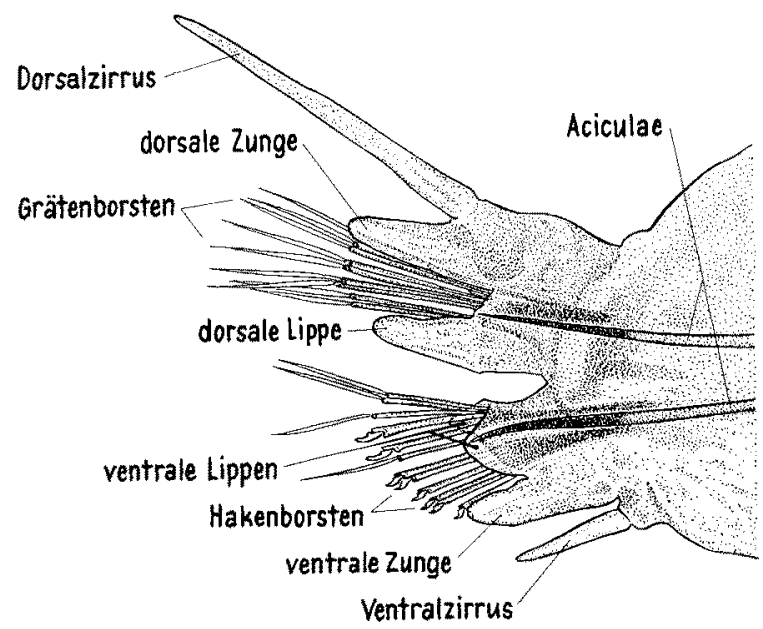

Abb. 4: Atokes Parapodium vom 32. Segment eines intakten $Q$ mit $40 \mu \mathrm{m}$ großen Oocyten (von hinten gesehen) 
und nach wie vor in sehr gutem. Zustand; Messerborsten waren an den Pp noch nicht hervorgetreten und Papillen am Pygidium, wie sie in der Normalentwicklung nur bei den $\delta$ Heteronereis, an kopflosen Fragmenten jedoch auch regelmäßig im 9 Geschlecht erscheinen, waren nicht einmal andeutungsweise vorhanden.

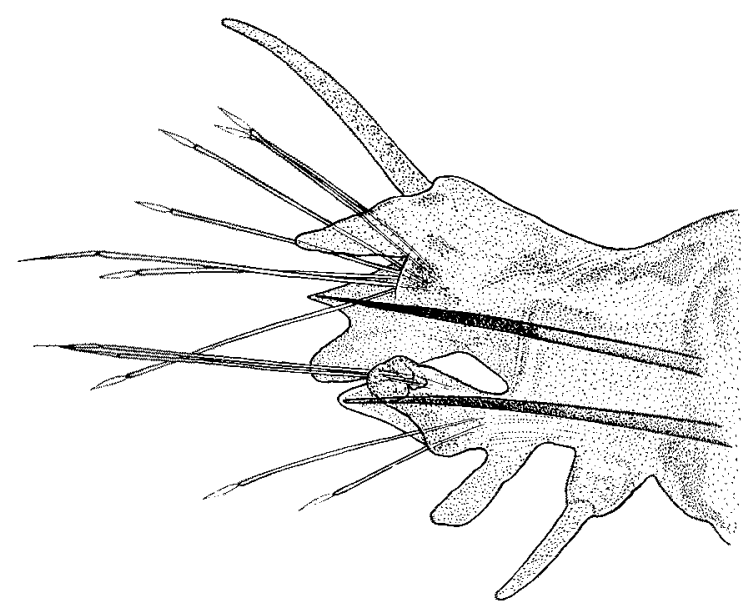

Abb. 5: 33. linkes Parapodium des in Abbildung 6 dargestellten Kontrollfragments

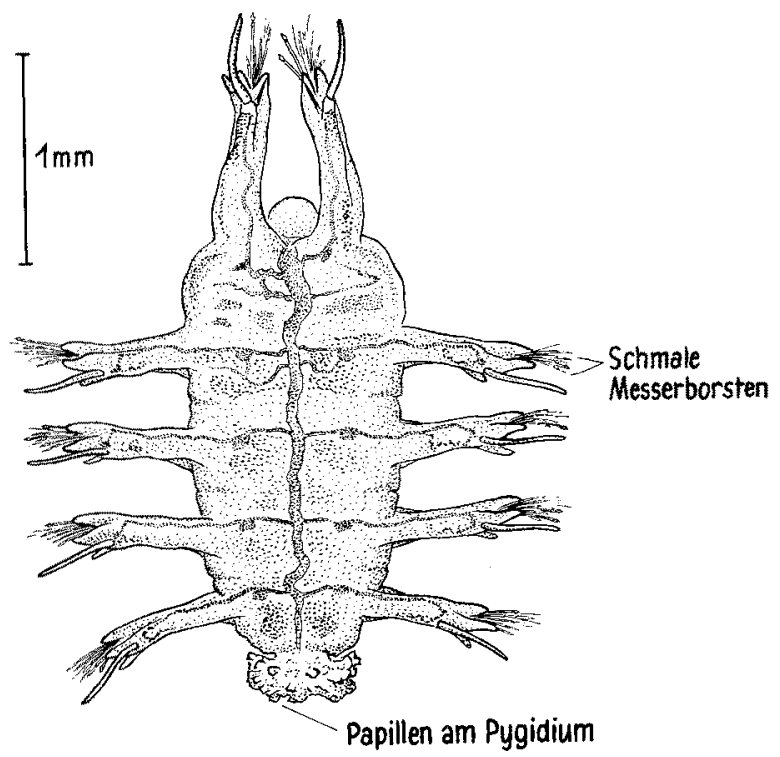

Abb. 6: Dasselbe Fragment wie in Abbildung 3 am 10. Versuchstag, d. h. nach 9tägiger Entwicklung als Kontrollfragment ohne Gehirnhormon. Der Beginn der bis zu 4 Tage dauernden Endphase, während der - außer Nekrosen - bis zum Tod keine äußeren Veränderungen mehr eintreten. steht unmittelbar bevor 
Demgegenüber bildete das $\mathrm{K}$ on trollf $\mathrm{ragment}$ weder einen gestreckten Regenerationskegel noch neue $\mathrm{PpS}$ und entwickelte auch keine Uriten; statt dessen erschienen am 7. Tag mehrere Papillen am Pygidium, die sich später blasig erweiterten (vgl. Abb. 6). Am 8. Tag traten, als eindeutiger Ansatz zu einer epitoken Entwicklung, an jedem Pp des KF zwischen den in der Mehrzahl noch vorhandenen Haken- und Grätenborsten in unregelmäßiger Verteilung 4-12 kleine Messerborsten hervor, deren Anhänge jedoch höchstens $60 \mu \mathrm{m}$ lang und $9 \mu \mathrm{m}$ breit waren (demgegenüber sind im normalen epitoken Pp 50-60 Messerborsten vorhanden, deren Anhänge etwa 3mal so lang und $3^{1 / 2}$ mal so breit sind); außerdem war an den Pp des KF die Basis der Ventralzirren jetzt deutlich sockelartig verdickt (vgl. Abb. 5). Am 9. Tag war eine gewisse Verlängerung und rostrocaudale Abplattung der Pp erkennbar und die Schäfte der


a

Abb. 7: Etwas mehr als halbwüchsige Oocyte(n), wie sie (a) nach einer Entwicklung ohne Gehirnhormon in einem Kontrollfragment am 10. Versuchstag (vergleichbar dem in Abbildung 6 dargestellten Stadium) rorlagen und (b) in einem intakten $q$ in Gegenwart von Gehirnhormon herangewachsen war

schmalen Messerborsten hatten ihre volle Länge erreicht; die tiefliegenden Chromatophoren waren nun restlos verschwunden und das Coelom des hierdurch hyalin wirkenden KF war deutlich etwas gebläht. Am 10. Tag stieß das KF (durch die Pygidialpapillen?) einige bis $100 \mu \mathrm{m}$ große Oocyten aus; sie waren alle abnorm transparent, weil sie nur relativ wenige, auf den perinukleären Bereich beschränkte Dotterkugeln enthielten. Die $\mathrm{Pp}$, an denen die meisten atoken Borsten jetzt ausgefallen waren, begannen zu dieser Zeit unregelmäßig und unkoordiniert einzelne Ruderschäge auszuführen, wobei sie - im Gegensatz zu den schräg rückwärts gerichteten, sich dachziegelartig deckenden epitoken $\mathrm{Pp}$ der normalen Heteronereis - in Ruhe nach atokem Modus seitlich abstanden; das KF blieb während dieser Pp-Bewegungen am Boden liegen und bewegte sich nicht von der Stelle. Die vom 7. bis zum 10. Tag in Ansätzen erkennbare epitoke Entwicklung des KF machte danach keinerlei Fortschritte mehr (vgl. Abb, 6). Am 12. Tag begann mit dem ersten Auftreten kleiner Nekroseherde (vor allem am Pygidium und seinen Papillen) die Endphase, in der sich das nunmehr krankhaft aussehende KF äußerlich nicht mehr veränderte und auf Berührung nur noch schwach reagierte; am 14. Tag war das KF tot. Zu diesem Zeitpunkt enthielt sein Coelom außer großen Mengen von Elaeocyten und einigen bereits in Auflösung begriffenen Oocyten 
30-40 hyaline, mit viel zu wenigen und teilweise unregelmäßig angeordneten Dotterkugeln ausgestattete Eizellen, die sich nach dem 10. Tag z. T. noch um $30 \mu \mathrm{m}$ bis $z u$ einem maximalen Durchmesser von $130 \mu \mathrm{m}$ vergrößert hatten (vgl. Abb. 7a); sie besaßen keine Andeutung der für nomale reife Eier typischen subkortikalen Gallert (Mucopolysaccharid)-Schicht und waren nicht befruchtungsfähig. Die Entwicklung der Pp war über das am 9. Tag erreichte Stadium, in dem epitoke Merkmale nur andeutungsweise vorhanden waren, nicht hinausgekommen.

Das Versuchsfragment lebte bis zum 20. Tag und somit 6 Tage länger als das KF. Nachdem seine caudale Regeneration - trotz der bis zum 19. Tag andauernden Behandlung mit täglich 3 juvenilen Prostomien - am 10. Tag zum Stillstand gekommen war, begann das VF am 11. Tag (d. h. 3 Tage später als das KF) mit dem Erscheinen von Messerborsten-Spitzen in die epitoke Entwicklung einzutreten. Die Messerborsten waren am 12. Tag zu voller Länge ausgewachsen und besaßen im Vergleich zu denjenigen des KF wesentlich größere, nämlich bis $150 \mu \mathrm{m}$ lange und $25 \mu \mathrm{m}$ breite Anhänge; auch waren beim VF pro $\mathrm{Pp}$ viel mehr Messerborsten ausgebildet als beim KF, nämlich 30-50, und diese ordneten sich am 13. Tag - wie bei einer normalen

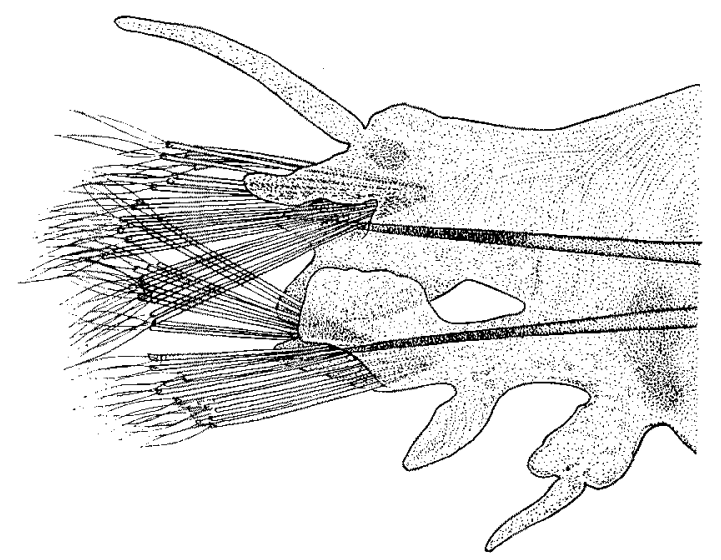

Abb. 8: 25, linkes Parapodium des in Abbildung 10 dargestellten Versuchsfragments

Heteronereis - zu regelmäßigen und breit gespreizten Fächern (Abb, 8), obwohl zu diesem Zeitpunkt noch nicht alle Haken- und Grätenborsten ausgefallen waren. Am 16. Tag erschien auch an den $P p$ der ersten beiden regenerierten Segmente je eine vollständige Garnitur von Messerborsten. Am 17. Tag waren an sämtlichen Pp fast alle atoken Borsten ausgefallen. Die Entwicklung der accessorischen epidermalen Lappen bzw. Lamellen, wie sie im Zuge der Epitokie an der Basis der Zirren und an den Lippen der Pp auswachsen, begann am 12. Tag und schritt bis zum 17. Tag fort; ihr Ergebnis reicht zwar nicht an den bei normalen reifen $q 0$, üblichen Ausprägungsgrad dieser epitoken Differenzierungen heran (Abb. 9), übertriff aber deutlich die diesbezüglichen Ansätze im zugehörigen KF (vgl. Abb. 8 und 5). Vom 15. Tag an hielt das VF seine nunmehr deutlich rostrocaudal abgeplatteten und verlängerten $\mathrm{Pp}$ - mit Ausnahme des nach vorn gestreckten ersten Paares - in der Ruhelage zunehmend schräg rückwärts 
gerichtet, so daß sich die Pp vom 17. Tag an (wenn auch nicht ganz so ausgeprägt wie beim normalen reifen O) gegenseitig dachziegelartig überdeckten (Abb. 10); diese Stellung der Pp spricht für eine weitgehend normale Ausbildung der im epitoken Zustand charakteristischen Transversalmuskeln. Dementsprechend begannen um diese Zeit die $\mathrm{Pp}$, zunächst vereinzelt und unkoordiniert, Ruderschläge auszuführen. Am 19. Tag bewegte sich das VF in gewissen Abständen eine Zeitlang koordiniert rudernd im Kreise, ohne sich jedoch vom Boden abheben zu können; dies spricht insofern für einen

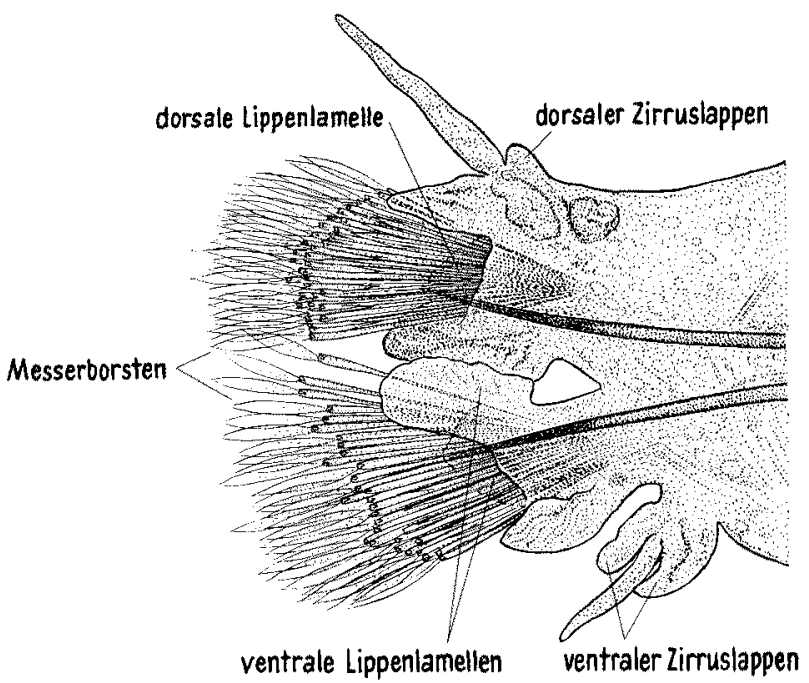

Abb. 9: Epitokes Parapodium vom 32. Segment einer intakten, reifen $q$ Heteronereis (zum Vergleich mit den in Abbildungen 8, 5, 4 dargestellten Parapodien)

Defekt in der epitoken Entwicklung, als nach früheren Beobachtungen auch kurze Fragmente, sofern sie mit Hilfe eines implantierten Prostomiums eine volle epitoke Ausprägung erreicht haben, grundsätzlich durchaus in der Lage sind, mittels Ruderbewegungen ihrer Pp längere Zeit frei durchs Wasser zu schwimmen. Die Oocyten des VF, welche in großer Anzahl durch die im Lauf der Entwicklung zunehmend durchscheinende Körperwand im Coelom erkennbar waren (Abb. 10), hatten am 12. Tag noch eine völlig unreife, normale Struktur (vgl. Abb. 7b) und waren schätzungsweise 110-120 $\mu \mathrm{m}$ groß; aber bereits am 13. Tag schienen sie deutlich vergrößert und in der Peripherie etwas aufgehellt zu sein. Vom 15. Tag an waren sie durch und durch hyalin, hatten eine schwach gelbliche Färbung angenommen und machten einen ausgewachsenen Eindruck; alles in allem sahen sie normalen reifen Eiern sehr ähnlich. Nachdem am 18. und 19. Tag eine Weiterentwicklung des VF in irgendeiner Hinsicht nicht mehr stattgefunden hatte, wurde ihm am 20. Tag, als mit dem Auftreten erster kleiner Nekroseherde sein Ende nahe schien, ein kleiner Teil seiner ca. 100-150 Eier zwecks genauer Untersuchung künstlich aus dem Coelom entnommen. Die Eier waren 170-185 $\mu \mathrm{m}$ und damit mindestens ebenso groß wie bei einem normalen $q$, vielleicht sogar von etwas übernormaler Größe; sie wiesen sowohl eine nahezu normale Dotterstruktur als auch 
eine voll entwickelte subkortikale Gallertschicht auf (Abb. 11a). Nach Besamung mit beweglichen, einer normalen $\&$ Heteronereis künstlich entnommenen Spermien schieden einige von thnen eine Gallerthülle aus; von diesen entwickelten sich vier binnen. 24 Stunden $\mathrm{zu} \pm$ wohlgestalteten Trochophora-Larven, welche allerdings nicht

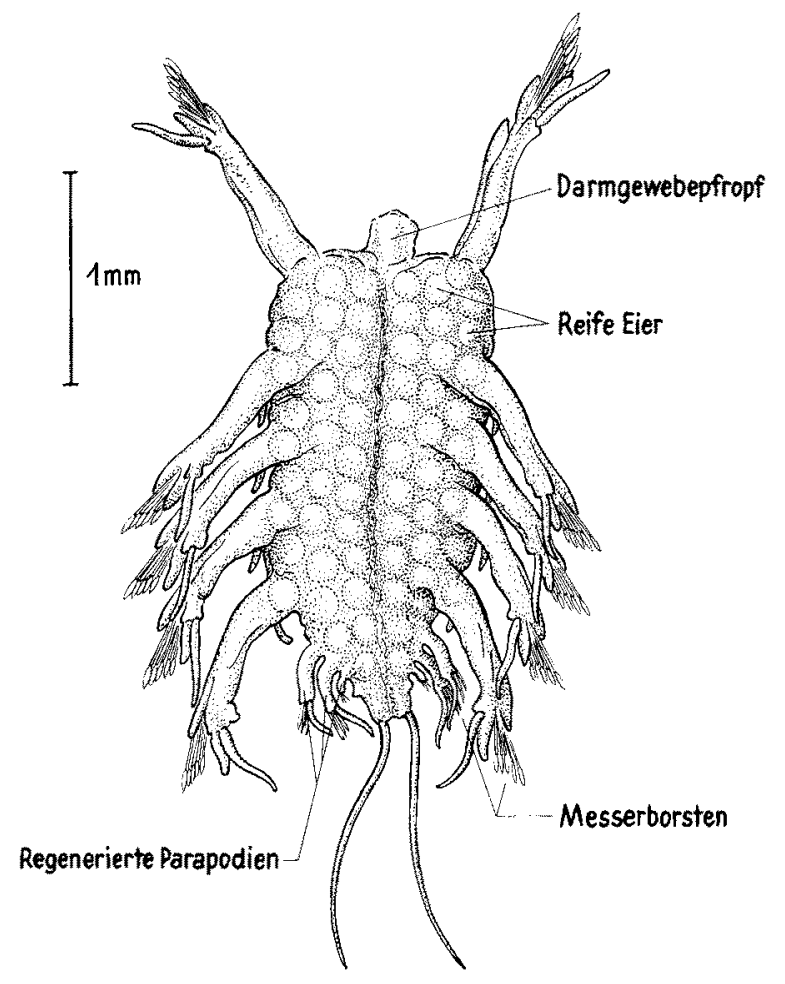

Abb. 10: Versuchsfragment (22,-26. PpS $+31 / 2$ regenerierte PpS), dessen Entwicklung im Text detailliert geschildert ist, am 20. Versuchstag. Das Versuchsfragment hatte 18 Tage vorher annähernd genauso wie das in Abbildung 3 dargestellte Kontrollfragment ausgesehen; infolge seiner vom 3. bis zum 19. Versuchstag andauernden Behandlung mit juvenilen Prostomien (vgl. Abb. 2) entwickelte es sich jedoch anders als die hormonfreie Kontrolle (vgl. Abb. 6)

schlüptten, sondern nur innerhalb der Eihülle rotierten. Da sich auch normale reife Eier nicht sehr gut entwickeln, wenn sie künstlich aus dem Coelom entnommen und besamt werden, scheint mir durch das vorliegende Ergebnis die prinzipielle Funktionsfähigkeit der vom VF gebildeten Eier hinreichend nachgewiesen. Nach der partiellen Eientnahme fixierte ich das VF am 20. Tag in Formol; nachdem eine Zeichnung von ihm angefertigt war (Abb. 10), wurden seine Pp an ihrer Basis vom Rumpf abgetrennt, um sie fotografieren, zeichnen (Abb. 8) und vermessen zu können.

Selbstverständlich weisen die Entwicklungsergebnisse in den 10 übrigen Versuchen eine gewisse Variabilität und damit Abweichungen von dem vorstehend geschilderten Fall hinsichtlich dieser oder jener Details auf. Insbesondere war der Epitokiegrad, den 
die KF erreichten, individuell merklich verschieden. So waren z. B. an den Pp einiger KF die Ventralzirrus-Lappen etwas stärker und die schmalen Messerborsten in etwas größerer Anzahl entwickelt, als in dem detailliert geschilderten Versuch; auch regenerierten einige $\mathrm{KF}$, ohne allerdings jemals einen ausgeprägten Regenerationskegel aufzubauen, in den ersten Tagen hinten ein- oder sogar beidseitig ein winziges $\mathrm{Pp}$ und/ oder kurze Uriten. Hinsichtlich der folgenden Kriterien ergaben sich jedoch keinerlei Überschneidungen $z$ wischen den Entwicklungsleistungen verschiedener VF auf der einen und denjenigen verschiedener KF auf der anderen Seite: Lebensdauer; Umfang und Qualität der caudalen Regeneration; Zeitpunkt des Beginns der epitoken Entwicklung; Entwicklung von Pygidialpapillen; Größe der Messerborsten-Anhänge; Größe, Struktur und Funktionsfähigkeit der Eier (letztere konnte allerdings nur einmal getestet

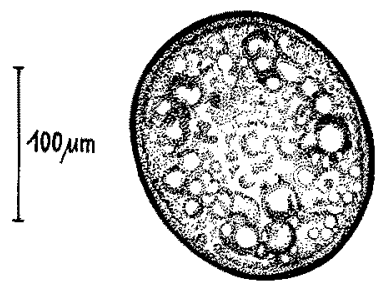

a

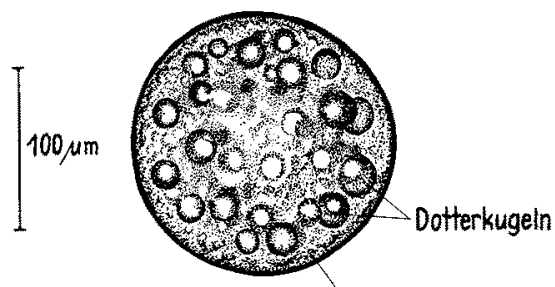

b
Subkortikale Gallertschicht aus Mucopolysacchariden

Abb. 11: Reifes, befruchtungs- und entwicklungsfähiges Ei; (a) aus dem in Abbildung 10 dargestellten Versuchsfragment (die etwas längliche Form des gezeichneten Exemplars ist nicht typisch), (b) aus einer normalen, reifen Heteronereis

werden). Uberdies kann beim Vergleich eines VF mit dem vom selben Spender- $q$ stammenden KF die individuelle Variabilität außer Betracht bleiben und es braucht statt dessen nur eine geringfügige regionale Verschiedenheit der in ihrer Entwicklungsleistung zu vergleichenden beiden Wurmstücke berücksichtigt zu werden. Somit ist festzustellen, daß sich durch das Hormon aus den Prostomien juvenilex Spender die Oogenese und die epitoke Entwicklung kopfloser Fragmente von jungen, zum Zeitpunkt der Zerteilung noch im vorkritischen Stadium befindlichen 우 entscheidend verbessern läßt; hierbei wird die beim normalen, schwärmreifen Heteronereis- $q$ vorliegende Norm bezüglich der Oogenese nahezu vollkommen, bezüglich der Epitokie allerdings nicht vollständig erreicht. Unabhängig vom Grad der Wirksamkeit entspricht die Art, in der das äußerlich applizierte Prostomium-Eluat die sexuelle Entwicklung beeinflußt, jedenfalls in ihrer Richtung genau der Wirkung, die ein implantiertes lebendes Prostomium auf ein vergleichbares Testfragment ausïbt.

In 5 weiteren Versuchen habe ich zum Vergleich mit derselben Methode die Wirkung von Prostomien \pm weit entwickelter $q q$ (mit Oocyten zwischen 50 und $140 \mu \mathrm{m}$ ) geprüft. Dabei gelang es in keinem Fall, die mit juvenilen Prostomien erzielten Entwicklungsleistungen überhaupt zu erreichen, geschweige denn zu übertreffen, weil die mit solchen älteren Prostomien kombinierten Versuchsfragmente stets vorzeitig degenerierten; vermutlich werden aus den Prostomien von bereits in Reifung begriffenen Spendern außer dem Gehirnhormon in viel höherem Maß, als dies bei juvenilen Prosto- 
mien der Fall ist, noch andere Substanzen freigesetzt, die bei ihrer äußeren Applikation in der sehr kleinen Wassermenge schädlich wirken.

Schließlich sollte noch untersucht werden, wie sich Fragmente von noch jüngeren 우, die überhaupt noch keine Geschlechtsprodukte im Coelom haben, unter dem Einfluß des Hormons aus juvenilen Prostomien entwickeln. Da sich die beiden hierzu verwendeten, aus 54 bzw. 56 PpS bestehenden Spendertiere im Lauf des Versuchs durch die Entwicklung ihrer Dorsalzirren als $\delta \delta \mathrm{zu}$ erkennen gaben, erfüllten diese zwei Versuche den ursprünglich beabsichtigten $Z$ weck nicht; ihre Ergebnisse weisen aber darauf hin, daß bei den $\hat{\sigma} \delta$ von Platynereis dumerilii prinzipiell ähnliche Verhältnisse wie bei den $q 9$ vorliegen. Auch bei diesen beiden $\hat{o} \hat{o}$ zeigten nämlich die VF größere Lebensdauer (25-27 Tage), mehr caudale Regeneration (21/2-4 PpS) und eine verzögerte, aber im Endeffekt weitergehende epitoke Entwicklung (breite Messerborsten, mittelgroße Zirruslappen) als die zugehörigen KF (13-18 Tage; O PpS; keine oder nur einzelne, sehr schmale Messerborsten und nur Andeutungen von Zirruslappen). Allerdings enthielten alle Fragmente, da bei den VF ebensowenig eine Spermatogenese stattfand wie bei den KF, bei Versuchsschluß im Coelom nur Elaeocyten.

\section{DISKUSSION}

Da aus fruheren Untersuchungen bekannt ist, daß eine nennenswerte und qualitativ normale caudale Regeneration nur in Gegenwart des juvenilen Gehirnhormons möglich ist, geht aus dem Befund, daß die VF eine solche Regenerationsleistung regelmäßig vollbringen, eindeutig hervor, daß das in Form von getrockneten und tiefgekühlt konservierten Prostomien äußerlich applizierte Hormon in das Testfragment eindringt und dort in prinzipiell normaler Weise, wenn auch sicher stark abgeschwächt, wirksam wird. Auch die Tatsache, daß durch eine täglich sich wiederholende Gegenwart solcher Prostomien während 8 von 24 Stunden die Lebensdauer kopfloser Fragmente signifikant verlängert und der Beginn ihrer epitoken Entwidklung verzögert wird, weist in die gleiche Richtung.

Die Spender der verwendeten Prostomien hatten durchweg noch keine frei im Coelom liegenden Keimzellen und waren, nach ihrer Segmentzahl und Größe zu urteilen, vom Beginn der geschlechtlichen Entwicklung noch mindestens mehrere Wochen entfernt. Man darf deshalb, auch wenn man eine spätere Förderung der Oogenese und der Epitokie durch ein besonderes Reifungshormon zunächst einmal für möglich hält und die von MüLler (1973) im Gehirn von P. dumeriliz erhobenen autoradiographischen Befunde in diesem Sinne deutet, wohl annehmen, daß ein solches Reifungshormon in den verwendeten Prostomien entweder überhaupt noch nicht oder nur in einer gemessen am juvenilen Gehirnhormon völlig unbedeutenden Menge enthalten ist; auch der von MülLER gefundene zeitliche Verlauf in der Vermehrung der in Frage stehenden großen NSZ führt, falls diese Zellen als Produzenten eines Reifungshormons in Frage kommen, zu demselben Schluß. Während bei einem lebend in ein Wurmfragment implantierten und dort fortlaufend Hormon sezernierenden Prostomium nicht auszuschließen ist, daß es nach einer gewissen Zeit von der Produktion des juvenilen Hormons auf eine Erzeugung von Reifungshormon umschaltet und dadurch die geschlecht- 
liche Reifung des Wirtsfragments in Gang bringt, erhält bei der in den vorliegenden Versuchen angewandten Methode das VF während seiner gesamten Entwicklungszeit mit Sicherheit, in Qualität und Quantität gleichbleibend, ausschließlich oder zumindest ganz überwiegend juveniles Hormon zugeführt.

$\mathrm{Da}$ die fortgesetzt mit juvenilem Gehirnhormon intermittierend behandelten VF zu einer so gut wie normalen Oogenese und zu einer verglichen mit den hormonfreien Kontrollen verbesserten epitoken Differenzierung befähigt waren, muß die Notwendigkeit eines besonderen, erst in einem fortgeschrittenen Stadium gebildeten prostomialen Reifungshormons für diese Entwicklungsprozesse verneint und damit wohl auch die Existenz eines solchen Hormons in der Normalentwicklung als äußerst unwahrscheinlich angesehen werden. Die Frage, ob sich $q 9$ mit noch kleinen Oocyten nach Abtrennung ihres Prostomiums darum nicht mehr normal und vollständig geschlechtlich entwickeln können, weil sie nun ein besonderes, vom juvenilen Hormon qualitativ verschiedenes Reifungshormon nicht mehr bekommen oder lediglich deshalb, weil auch für die Reifung ein gewisser Spiegel an gewöhnlichem juvenilem Gehirnhormon erforderlich ist und dieser ohne Prostomium nicht mehr aufrecht erhalten werden kann, läßt sich nach den vorliegenden Ergebnissen in dem letztgenannten Sinne beantworten. Man darf deshalb annehmen, daß die großen, synthetisch aktiven NSZ im anteriodorsalen und im posterioventralen Bereich des Gehirns, für die Müller eine drastische zahlenmäßige Zunahme im Verlauf der fortschreitenden geschlechtlichen Entwicklung nachgewiesen hat, ein Neurosekret produzieren, welches nicht in die Oogenese und in die epitoke Morphogenese als solche eingreift, sondern vielmehr irgendwelche Funktionen der reifen Heteronereis vorbereitet, auslöst oder kontrolliert. Da die VF in den vorliegenden Experimenten - anders als viele ebenso kurze Fragmente, die mit einem implantierten lebenden Prostomium reif wurden - trotz augenscheinlich ausreichender anatomischer Voraussetzungen nicht schwimmfähig waren, könnte man an eine Wirkung des betreffenden Inkrets auf die Muskeltätigkeit beim Schwimmen und beim Ausstoß der Geschlechtsprodukte denken; auch eine Rolle als Pheromon, mit dem sich die Geschlechter über eine gewisse Distanz anlocken und synchronisieren, käme beispielsweise in Frage. Da nach den Befunden von Durchon \& Porchet (1971) der Spiegel des juvenilen Gehirnhormons während der geschlechtlichen Entwicklung etwa in dem gleichen zeitlichen Verlauf abnimmt, in dem nach Müller (1973) die Anzahl der NSZ im Gehirn zunimmt, liegt es nahe anzunehmen, daß es sich bei diesen NSZ um Neurone handelt, die vorher in einem hinsichtlich Größe und Färbbarkeit wenig auffälligen Zustand solange juveniles Hormon produziert haben, bis sie sich im Verlauf der Reifung unter Zunahme ihrer Größe nach und nach auf die Produktion eines anderen, gut anfärbbaren Neurosekrets umstellten.

Auf Grund früherer Ergebnisse, wie ich sie z. B. nach Implantation von Larvenköpfen oder nach Bestrahlung des Gehirns mit UV erhalten hatte (HauENschrLd 1966), begründete ich eine Hypothese, derzufolge alle im Zuge der geschlechtlichen Entwicklung in bestimmter zeitlicher Sequenz ablaufenden Prozesse durch einen langsam absinkenden Gehirnhormonspiegel so gesteuert werden sollten, daß die einleitenden Vorgänge (z. B. langsames Oocytenwachstum) an einen relativ höheren, die abschließenden (z. B. Eireifung) dagegen an einen relativ niedrigeren Hormonspiegel gebunden sind; mit anderen Worten, jeder Vorgang sollte nur bei einer bestimmten Hormonkonzen- 
tration in Gang kommen und normal ablaufen können. Von den beiden sich gegenseitig ausschließenden Prozessen Regeneration und Reifung sollte nach dieser Vorstellung die Neubildung von Segmenten nur in Gegenwart einer ziemlich großen Hormonmenge, die geschlechtliche Entwicklung dagegen nur bei deutlich geringeren Hormonquantitäten möglich sein. Auf Grund der vorliegenden Ergebnisse bedarf diese Auffassung wahrscheinlich einer gewissen Korrektur. Die VF waren ja vom 3. Tag bis zur Vollendung ihrer sexuellen Reifung einer nicht nur qualitativ, sondern auch quantitativ völlig gleichbleibenden Hormonbehandlung unterworfen. Dennoch reagierten sie in den ersten 8-10 Tagen mit caudaler Regeneration und schalteten dann, obwohl sich an der angebotenen Menge Gehirnhormon nichts änderte, sozusagen spontan auf Geschlechtsreifung um. Es ist allerdings nicht auszuschließen, daß in einem Fragment eine gewisse Menge Gehirnhormon, die bereits vor seiner Isolierung von dem zugehörigen Prostomium dorthin gelangt war, tagelang in wirksamer Form erhalten bleibt, bevor sie restlos inaktiviert, abgebaut oder ausgeschieden ist; in diesem Fall wäre, zusammen mit dem äußerlich zugeführten Hormon, der effektive Hormonspiegel anfangs doch etwas höher als später. Sollte dies zutreffen, könnte die Beschränkung der Regenerationsfähigkeit des VF auf die erste Versuchswoche in der Tat darauf beruhen, daß nur in dieser Zeit durch Summierung des überkommenen Rest-Hormons mit dem von außen zugeführten Hormon die für eine Segmentneubildung erforderliche Schwelle überschritten wird, wohingegen dies später, nach dem Verschwinden des Rest-Hormons, nicht mehr der Fall ist. Die in der zweiten Versuchshälfte trotz gleichbleibenden Hormonangebots normal verlaufende Oogenese, deren einzelne Phasen auch an verschiedene Hormonkonzentrationen geknüptt sein sollten (vgl. DuRchon \& Porchet 1971), dürfte sich jedoch nicht ohne weiteres in dem gleichen Sinne interpretieren lassen. Vorbehaltlich einer Klärung der Frage, nach welcher Zeit das bei Versuchsbeginn in VF und KF gleichermaßen vorhandene Rest-Hormon verschwunden ist, wird man deshalb zur Deutung der vorliegenden Ergebnisse vielleicht auch eine im Laufe des Versuchs fortschreitende autonome Anderung der Reaktionsweise des Fragments gegenüber dem Hormon ins Auge fassen müssen; eine solche Veränderung im reagierenden System könnte durch eine einmalige Absenkung des Hormonspiegels zu Versuchsbeginn in Gang gesetzt werden und sich danach selbständig fortsetzen. Offen bleibt zunächst, ob man hierbei an eine im Versuchsverlauf allmählich abnehmende Durchlässigkeit bzw. Aufnahmebereitschaft des VF-Integuments für das von außen zugeführte Hormon oder an eine veränderte Reaktion der an Regeneration und geschlechtlicher Entwicklung unmittelbar beteiligten Zellen und Gewebe selbst (etwa im Sinne einer Schwellenerhöhung) denken soll. In bezug auf die Regenerationsfähigkeit wird das letztere durch einen Befund von Hofmann (1966) nahegelegt, demzufolge Fragmente, denen erst 5 (bzw. 6) Tage nach ihrer Isolierung ein lebendes Prostomium ins Coelom gesteckt wird, nur noch vermindert (bzw. gar nicht mehr) zur Neubildung von Segmenten befähigt sind.

In allen Versuchen erreichten die VF zwischen dem 15. und 20. Tag den Höhepunkt ihrer geschlechtlichen Entwicklung, sowohl in bezug auf die Eireifung als auch hinsichtlich ihrer Epitokie. Dies erscheint insofern bemerkenswert, als in den unter zyklischen Belichtungsbedingungen gehaltenen Platynereis-Zuchten die meisten reifen Heteronereis nach einer langjährigen Statistik 18 Tage nach dem als auslösend erkann- 
ten Ereignis, nämlich dem Übergang von nächtlicher Belichtung zu nächtlicher Dunkelheit, auftraten. Bei der vorzeitigen Auslösung der geschlechtlichen Reifung junger o우 durch UV-Bestrahlung des Gehirns dauerte es, bei allerdings beträchtlicher Streuung, im Mittel 19 Tage bis zum Erreichen des schwärmreifen Heteronereis-Stadiums. Ebenso ergab sich auch für +-Fragmente mit außen aufgepfropftem Larvenkopf eine ganz ähnliche mittlere Entwicklungsdauer bis zum Eintritt der vollen Geschlechtsreife, nämlich 20 Tage (Hauenschild 1966). Es scheint demnach, daß die Reifung der $q 9+$ von $P$. dumerilii endgültig erst durch eine einmalige Herabsetzung der verfügbaren Gehirnhormon-Menge in Gang kommt und anschließend, unabhängig von der jeweiligen Ursache der Hormonspiegel-Senkung, innerhalb einer ziemlich konstanten Frist abläuft.

\section{ZUSAMMENFASSUNG}

1. Ein kurzes Fragment von Platynereis dumerilii, das vom Prostomium als seiner natürlichen Gehirnhormon-Quelle abgeschnitten ist, vermag ein normales Regenerat an seiner hinteren Schnittfläche, wie es intakte Tiere nach Verlust ihres Hinterendes stets zu bilden pflegen, nicht hervorzubringen. Es erlangt diese Fähigkeit jedoch wieder, wenn es täglich 8 Stunden lang in einem kleinen Tropfen mit drei getrockneten Prostomien von juvenilen Spendern kombiniert wird. Demnach nimmt das Fragment aus dem Außenmedium Gehirnhormon, welches nur aus den zerfallenden Prostomien stammen kann, in wirksamer Form und in einer für die Regeneration ausreichenden Menge auf.

2. Stammt das Fragment von einem $\uparrow$ mit erst $40 \mu \mathrm{m}$ großen Oocyten, so ermöglichen die zugesetzten toten Prostomien juveniler Spender außerdem eine fast normale Oogenese, wie sie auf diesem Stadium in völliger Abwesenheit von Gehirnhormon niemals stattfindet sowie einen Grad von Epitokie, welcher über denjenigen eines unbehandelten Kontrollfragments deutlich hinausgeht. Die Tatsache, daß die Entwicklung der Oocyten im hormonfreien Kontrollfragment einen abnormen Verlauf nimmt, beruht demnach nicht auf dem Fehlen eines besonderen, nur in einem fortgeschrittenen Stadium der Geschlechtsreife gebildeten „Reifungshormons", sondern nur auf dem vollständigen Mangel an dem "gewöhnlichen" Gehirnhormon, das bereits im juvenilen Stadium produziert wird.

3. Die von MüLler (1973) im Gehirn von $P$. dumerilii nachgewiesenen und autoradiographisch als synthetisch aktiv gekennzeichneten neurosekretorischen Zellen, deren Anzahl im Verlauf der geschlechtlichen Entwicklung stark zunimmt, kommen somit nicht für die Produktion eines morphogenetisch wirksamen, spezifischen „Reifungshormons" in Betracht. Sie müssen vielmehr eine andere Funktion, etwa im Zusammenhang mit dem Schwärmen der reifen Heteronereis, haben. Es könnte sich bei ihnen um die Zellen handeln, die fruher das juvenile Gehirnhormon produziert und die nach dem Erlöschen dieser Funktion während der Geschlechtsreifung ihr Aussehen und ihre inkretorische Tätigkeit geändert haben.

4. Die Tatsache, daß ein Fragment, dem während der ganzen Versuchszeit täglich dieselbe Dosis Gehirnhormon zugeführt wird, anfangs mit Regeneration, später jedoch mit geschlechtlicher Reifung antwortet, obwohl nach den bisherigen Vorstellungen 
der erstgenannte Vorgang an einen hohen und der letztere an einen niedrigen Hormonspiegel gebunden sein sollte, weist auf die Möglichkeit hin, daß auch die Art und Weise, in der das Fragment auf das Hormon anspricht, im Verlauf des Versuchs ihrerseits einer Veränderung unterliegt; davon könnten die an der Hormonaufnahme mitwirkenden und/oder die an Reifung bzw. Regeneration unmittelbar beteiligten Gewebe betroffen sein.

Danksagungen. Für technische Assistenz danke ich Frl. A. Ende, für die Anfertigung der Zeichnungen Herrn G. LINHARDT. Einige in den Untersuchungen verwendete Geräte wurden mir dankenswerterweise von der DFG als Leihgaben zur Verfügung gestellt.

\section{ZITIERTE LITERATUR}

Durchon, M., 1948. Épitoquie expérimentale chez deux polychètes: Perinereis cultrifera et Nereis irrorata. C. r. hebd. Séanc. Acad. Sci., Paris 227, 157-158.

- 1952. Recherches expérimentales sur deux aspects de la reproduction chez les Annélides Polychètes: L'épitoquie et la stolonisation. Annl. Soc. nat. Zool. Biol. anim. (Sér. 11) 14, 119-206.

- \& Porchet, M., 1971. Premières données quantitatives sur l'activité endocrine du cerveau des Néréidiens au cours de leur cycle sexuel. Gen. comp. Endocrinol. 16, 555-565.

Hauenschild, C., 1956. Hormonale Hemmung der Geschlechtsreife und Metamorphose bei dem Polychaeten Platynereis dumerilii. Z. Naturf. 11 b, 125-132.

- 1960. Abhängigkeit der Regenerationsleistung von der inneren Sekretion im Prostomium bei Platynereis dumerilii. Z. Naturf. 15 b, 52-55.

- 1965. Hormone bei Nereiden und anderen niederen Wirbellosen. Zool. Jb. (Allg. Zool. Physiol. Tiere) $71,511-544$.

- 1966. Der hormonale Einfluß des Gehirns auf die sexuelle Entwicklung bei dem Polychaeten Platynereis dumerilii. Gen. comp. Endocrinol. 6, 26-73.

- 1974. Endokrine Beeinflussung der geschlechtlichen Entwicklung einiger Polychaeten. Fortschr. Zool. 22 (2-3), 75-92.

- \& Fischer, A, 1969. Platynereis dimerilii. Mikroskopische Anatomie, Fortpflanzung, Entwicklung. Fischer, Stuttgart, 55 pp. (Großes zool. Prakt. $10 \mathrm{~b}$ ).

HofmanN, D. K., 1966. Untersuchungen zur Regeneration des Hinterendes bei Platynereis dumerilii. Zool. Jb. (Allg. Zool. Physiol. Tiere) 72, 374-430.

MüLLER, W. A., 1973. Autoradiographische Studien über die synthetische Aktivität neurosekretorischer Zellen im Gehirn von Platynereis dumerilii während der sexuellen Entwicklung und Regeneration. Z. Zellforsch. mikrosk. Anat. 139, 487-510.

PorcheT, M., 1967. Rôle des ovocytes submatures dans l'arrêt de l'inhibition cérébrale chez Perinereis cultrifera. C. r. hebd. Séanc. Acad. Sci., Paris 265, 1394-1396.

Anschrift des Autors:

Prof. Dr. C. Hauenschild

Zoologisches Institut

Technische Universität Carolo-Wilhelmina

33 Braunschweig

Pockelstraße 10a

Bundesrepublik Deutschland 